\title{
THE NOETHERIAN PROPERTY IN RINGS OF INTEGER-VALUED POLYNOMIALS
}

\author{
ROBERT GILMER, WILLIAM HEINZER, AND DAVID LANTZ
}

\begin{abstract}
Let $D$ be a Noetherian domain, $D^{\prime}$ its integral closure, and $\operatorname{Int}(D)$ its ring of integer-valued polynomials in a single variable. It is shown that, if $D^{\prime}$ has a maximal ideal $M^{\prime}$ of height one for which $D^{\prime} / M^{\prime}$ is a finite field, then $\operatorname{Int}(D)$ is not Noetherian; indeed, if $M^{\prime}$ is the only maximal ideal of $D^{\prime}$ lying over $M^{\prime} \cap D$, then not even $\operatorname{Spec}(\operatorname{Int}(D))$ is Noetherian. On the other hand, if every height-one maximal ideal of $D^{\prime}$ has infinite residue field, then a sufficient condition for $\operatorname{Int}(D)$ to be Noetherian is that the global transform of $D$ is a finitely generated $D$-module.
\end{abstract}

\section{INTRODUCTION}

Throughout this paper $D$ denotes an integral domain (commutative, with unity), $K$ denotes its field of fractions, and $X$ denotes an indeterminate. The ring of integer-valued polynomials of $D$ (in the single indeterminate $X$ ) is

$$
\operatorname{Int}(D)=\{f(X) \in K[X]: \forall a \in D, f(a) \in D\} .
$$

The rings of integer-valued polynomials on various domains have been studied at least since the 1919 articles of Ostrowski [O] and Pólya [P]. Even at that time it was known that the ring of integer-valued polynomials, $\operatorname{Int}(\mathbb{Z})$, is generated as a $\mathbb{Z}$-module by the binomial coefficient polynomials

$$
\left(\begin{array}{l}
X \\
n
\end{array}\right)=X(X-1) \cdots(X-n+1) / n !
$$

for $n=0,1,2, \ldots$ The $\operatorname{ring} R=\operatorname{Int}(\mathbb{Z})$ is an interesting example of a nonNoetherian ring that "occurs in nature": It follows from [Ca1, Corollary 1.3] that $\operatorname{dim}(R)=2$, and Brizolis [B] showed that $R$ is a Prüfer domain, so $R$ is not Noetherian. (There are perhaps more direct ways of reaching the same conclusion-cf. [Ch2, p. 1]-but we use a similar method below.) In the present paper we investigate the Noetherian property of the $\operatorname{rings} \operatorname{Int}(D)$, and of their spectra. We will need the following well-known result.

Received by the editors September 21, 1990 and, in revised form, March 27, 1991.

1980 Mathematics Subject Classification (1985 Revision). Primary 13G05, 13E05, 13 B99.

Key words and phrases. Rings of integer-valued polynomials, Noetherian domain, Noetherian spectrum, Prüfer domain, Krull domain, integral closure, global transform.

Heinzer gratefully acknowledges the support of National Science Foundation grant DMS 8800762 and Lantz the hospitality of Purdue University while this work was done. 
Fact 1.1 [SSY, Theorem 2; Ch1, Proposition 2.2]. If $D$ is a Noetherian domain, then $\operatorname{Int}(D) \neq D[X]$ iff there is a maximal ideal $M$ of $D$ that is an associated prime of a principal ideal $a D$ (i.e., for some $b$ in $D, M=a D: b$ ) and such that the residue field $D / M$ is finite.

We prove the following statements for a Noetherian domain $D$ with integral closure $D^{\prime}$ : If $D^{\prime}$ has a maximal ideal $M^{\prime}$ of height one for which $D^{\prime} / M^{\prime}$ is finite, then $\operatorname{Int}(D)$ is not Noetherian. (In fact, if $M^{\prime}$ is the only maximal ideal of $D^{\prime}$ lying over $M^{\prime} \cap D$, then not even $\operatorname{Spec}(\operatorname{Int}(D))$ is Noetherian.) In particular, if $\operatorname{dim}(D)=1$, then $\operatorname{Int}(D)$ is Noetherian iff $\operatorname{Int}(D)=D[X]$. On the other hand, if $D^{\prime}$ has no height-one maximal ideal with finite residue field, then a sufficient condition for $\operatorname{Int}(D)$ to be Noetherian is that the global transform of $D$ (in the sense of $[\mathrm{Mj}]$ ) is a finitely generated module over $D$. We also provide an example of a two-dimensional Noetherian domain $D$ for which, for each maximal ideal $M$ of $D, \operatorname{Int}\left(D_{M}\right)$ is Noetherian, but $\operatorname{Int}(D)$ is not Noetherian.

In terms of the spectrum of $\operatorname{Int}(D)$, we prove in Theorem 3.1 that for a one-dimensional local domain $(D, M)$ with finite residue field, $\operatorname{Spec}(\operatorname{Int}(D))$ is Noetherian if and only if $D^{\prime}$ has more than one maximal ideal.

We consistently use ' to denote integral closure of a domain (in its field of fractions). When no confusion is likely, we write $E$ in place of $\operatorname{Int}(D)$. The spectrum (i.e., the set of proper prime ideals) and the maximal spectrum of a domain $D$ are denoted $\operatorname{Spec}(D)$ and $\operatorname{Mspec}(D)$, respectively. The terms "local" and "semilocal" include "Noetherian". We use < to denote proper set inclusion.

Let $a$ be any element of $D$. Then the evaluation map $\varphi: E \rightarrow D$ defined by $\varphi(p)=p(a)$ is a retraction of $D$-algebras. Its kernel is the prime ideal $(X-a) K[X] \cap E$. Since $D$ is an algebraic retract of $E, D$ is "ideally closed" in $E$-i.e., for each ideal $I$ of $D, I E \cap D=I$. In particular, if $E$ is Noetherian, then $D$ is Noetherian. Thus, most of our results lose nothing with the addition of a Noetherian hypothesis on $D$. Moreover, if $S$ is a multiplicatively closed subset of $D$, then the equality $(\operatorname{Int}(D))_{S}=\operatorname{Int}\left(D_{S}\right)$ holds if $D$ is Noetherian [CC, p. 303], but not in general, as is shown in [G] by the construction of an almost Dedekind domain $D$ for which $\operatorname{Int}(D)$ is not a Prüfer domain.

\section{THE NOETHERIAN PROPERTY IN $\operatorname{Int}(D)$}

It turns out that the domain $\operatorname{Int}\left(D^{\prime}\right)$ plays a significant role in our work on the problem of determining conditions under which $\operatorname{Int}(D)$ is Noetherian, particularly in the case where $D$ is one-dimensional. In general, if $D_{1}$ is a subring of an integral domain $D_{2}$, it need not be true that $\operatorname{Int}\left(D_{1}\right)$ is contained in $\operatorname{Int}\left(D_{2}\right)$. (They are, of course, both subrings of $K[X]$, where $K$ is the field of fractions of $D_{2}$.) For example, if $k$ is the field of two elements, $t$ is an indeterminate, $D_{1}=k\left[\left[t^{2}, t^{3}\right]\right]$, and $D_{2}=k[[t]]=\left(D_{1}\right)^{\prime}$, then $\left[\left(1 / t^{2}\right) X(X+1)\right]^{2}$ is in $\operatorname{Int}\left(D_{1}\right)$ but not in $\operatorname{Int}\left(D_{2}\right)$. This phenomenon forced us to exercise some care, especially in our notation: If $E=\operatorname{Int}(D)$, we need not have $E^{\prime}=\operatorname{Int}\left(D^{\prime}\right)$. (In fact, in Proposition 6.1, we give necessary and sufficient conditions on a one-dimensional Noetherian domain $D$ in order that $\operatorname{Int}(D) \subseteq \operatorname{Int}\left(D^{\prime}\right)$.) Thus Proposition 2.2 below is reassuring. Recall that a 
transversal of a family $\mathcal{N}$ of sets is a set $\left\{s_{N}: N \in \mathscr{N}\right\}$ such that $s_{N} \in N$ for each $N$ in $\mathscr{N}$.

Remark 2.1. Let $R$ and $S$ be subrings of a larger ring (commutative, with unity), and let $\mathscr{N}$ be a family of multiplicatively closed subsets of $R \cap S$ such that every transversal of $\mathcal{N}$ generates the unit ideal in $R$. Then an element $s$ of $S$ is integral over $R$ iff, for each $N$ in $\mathscr{N}$, the image of $s$ in $S_{N}$ is integral over $R_{N}$.

Proof. If $s$ in $S$ is integral over $R$, it is clear that the image of $s$ in $S_{N}$ is integral over $R_{N}$. Conversely, if the local condition is satisfied by $s$, then for each $N$ in $\mathscr{N}, s$ is a root of a polynomial $p_{N}(X)$ in $R[X]$ with leading coefficient in $N$. Since a finite set of these leading coefficients generates the unit ideal in $R$, the ideal in $R[X]$ generated by the corresponding polynomials $p_{N}(X)$ contains a monic polynomial, of which $s$ is a root.

Proposition 2.2. Let $D$ be a Noetherian domain, with field of fractions $K$ and integral closure $D^{\prime}$. Then the ring $\operatorname{Int}\left(D, D^{\prime}\right)$ of polynomials $f(X)$ in $K[X]$ for which $f(D) \subseteq D^{\prime}$ is the integral closure of $\operatorname{Int}(D)$.

Proof. Since $\operatorname{Int}\left(D, D^{\prime}\right)$ is contained in the field of fractions $K(X)$ of $\operatorname{Int}(D)$, it is enough to show that $\operatorname{Int}\left(D, D^{\prime}\right)$ is integrally closed and integral over $\operatorname{Int}(D)$. But it is immediate that, for any subset $S$ of an integrally closed domain $R$, the ring $\operatorname{Int}(S, R)$ of polynomials $f(X)$ with coefficients in the field of fractions of $R$ and for which $f(S) \subseteq R$ is integrally closed (since its integral closure is contained in the ring of polynomials over the field of fractions of $R$, and if $g(X)$ is a polynomial over the field of fractions of $R$ integral over $\operatorname{Int}(S, R)$, then for each $s$ in $S, g(s)$ is integral over $R$ and hence in $R)$.

To see that $\operatorname{Int}\left(D, D^{\prime}\right)$ is integral over $\operatorname{Int}(D)$, let $f(X)$ be an element of $\operatorname{Int}\left(D, D^{\prime}\right)$. It suffices to show that, for each maximal ideal $M^{\prime}$ of $D^{\prime}$, there is an element $s$ of $D^{\prime}-M^{\prime}$ for which $s f$ is integral over $\operatorname{Int}\left(D_{M^{\prime} \cap D}\right.$ ) (for then, possibly with a different $s, s f$ will be integral over $\operatorname{Int}(D)$, and since the integral closure $\operatorname{Int}(D)^{\prime}$ of $\operatorname{Int}(D)$ is a module over $D^{\prime}$, it will follow that $\left.f \in \operatorname{Int}(D)^{\prime}\right)$. So take the maximal ideal $M^{\prime}$ of $D^{\prime}$, and assume $D$ is local with maximal ideal $M^{\prime} \cap D$.

Suppose first that $M^{\prime}$ has height greater than one. Then by Proposition 4.1, $f \in \bigcap\left\{D_{P^{\prime}}^{\prime}[X]: P^{\prime} \subseteq M^{\prime}, \operatorname{ht}\left(P^{\prime}\right)=1\right\}=D_{M^{\prime}}^{\prime}[X]$, so there is an element $s$ of $D^{\prime}-M^{\prime}$ for which $s f \in D^{\prime}[X]$, i.e., $s f$ is integral over $D[X] \subseteq \operatorname{Int}(D)$. Similarly, if $M^{\prime}$ has height one and $D^{\prime} / M^{\prime}$ is infinite, then again $f \in D_{M^{\prime}}^{\prime}[X]$, so there is an $s \in D^{\prime}-M^{\prime}$ such that $s f$ is integral over $\operatorname{Int}(D)$.

Now suppose $M^{\prime}$ has height one and $D^{\prime} / M^{\prime}$ is finite. Since $f(D) \subseteq D^{\prime} \cap$ $(1 / d) D$, where $d$ is a common denominator for the coefficients of $f$, there is a ring $R$ contained in $D^{\prime}$, finitely generated over $D$, for which $f(D) \subseteq R$ and $M^{\prime}$ is the only maximal ideal of $D^{\prime}$ lying over $M^{\prime} \cap R=N$. Let $C=D:{ }_{R} R$, $s$ be an element of $R-N$ for which $s\left(C R_{N} \cap R\right) \subseteq C$ (such an element exists because $R$ is Noetherian, so that $C R_{N} \cap R$ is finitely generated), $q$ be the cardinality of $R / N$, and $n$ be a positive integer for which $N^{n} \subseteq C R_{N} \cap R$ (such an integer exists because $\operatorname{ht}(N)=1$, so that $C R_{N} \cap R$ is $N$-primary). Then for each $a$ in $D,(s f(a))^{q}-s f(a) \in N$, so $\left((s f(a))^{q}-s f(a)\right)^{n} \in C R_{N} \cap R$, 
so

$$
\begin{aligned}
\left((s f(a))^{q}-s f(a)\right)^{n+1} & =s\left(s^{q-1} f(a)^{q}-f(a)\right)\left((s f(a))^{q}-s f(a)\right)^{n} \\
& \in s\left(C R_{N} \cap R\right) \subseteq C \subseteq D,
\end{aligned}
$$

so $g=\left((s f)^{q}-s f\right)^{n+1} \in \operatorname{Int}(D)$. Thus $s f$ is a root of the monic polynomial $\left(Y^{q}-Y\right)^{n+1}-g$ over $\operatorname{Int}(D)$.

Theorem 2.3. Let $D$ be a Noetherian domain for which $\operatorname{Int}(D)$ is also Noetherian. Then for every maximal ideal $M^{\prime}$ of the integral closure $D^{\prime}$ of $D$, either $M^{\prime}$ has height greater than one, or else the residue field $D^{\prime} / M^{\prime}$ is infinite.

Proof. Assume by way of contradiction that a maximal ideal $M^{\prime}$ of $D^{\prime}$ has height one and finite residue field; set $M=M^{\prime} \cap D$. Then by localizing $D$ and $D^{\prime}$ at $D \backslash M$, we preserve the hypothesis, so we may assume $(D, M)$ is local. Next, adjoining to $D$ an element of $M^{\prime}$ not in any of the other maximal ideals of $D^{\prime}$ (these are finite in number $[\mathrm{Na},(33.10)]$ ), we form a ring $R$, finitely generated as a $D$-module, for which $D^{\prime}=R^{\prime}$ and $M^{\prime}$ is the only maximal ideal of $D^{\prime}$ lying over $M^{\prime} \cap R=N$. Since the nonzero conductor of $R$ into $D$ also multiplies $\operatorname{Int}(R)$ into $\operatorname{Int}(D)$, the compositum $E[\operatorname{Int}(R)]$ of $E=\operatorname{Int}(D)$ and $\operatorname{Int}(R)$ is (contained in) a finitely generated module over $\operatorname{Int}(D)$ and hence is also Noetherian. Localizing at the multiplicative set $R \backslash N$, we see that $(E[\operatorname{Int}(R)])_{R \backslash N}=E\left[(\operatorname{Int}(R))_{R \backslash N}\right]=E\left[\operatorname{Int}\left(R_{N}\right)\right]$ is also Noetherian, and that the integral closure of $R_{N}$ is $D_{M^{\prime}}^{\prime}$, a discrete rank-one valuation domain. By Proposition 2.2, $\operatorname{Int}\left(D_{M^{\prime}}^{\prime}\right)$ is contained in the integral closure $\left(\operatorname{Int}\left(R_{N}\right)\right)^{\prime}$ of $\operatorname{Int}\left(R_{N}\right)$ and hence in $\left(E\left[\operatorname{Int}\left(R_{N}\right)\right]\right)^{\prime}$. On the one hand, as the integral closure of a Noetherian domain, $\left(E\left[\operatorname{Int}\left(R_{N}\right)\right]\right)^{\prime}$ is Krull. On the other hand, as an overring of $\operatorname{Int}\left(D_{M^{\prime}}^{\prime}\right)$, which is a Prüfer domain by, for example, [Ch2, Corollaire 6.5], $\left(E\left[\operatorname{Int}\left(R_{N}\right)\right]\right)^{\prime}$ is also a Prüfer domain; so it is Dedekind and hence one dimensional. However, since both $E=\operatorname{Int}(D)$ and $\operatorname{Int}\left(R_{N}\right)$ are contained in the ranktwo valuation domain $V=\left\{f \in K[X]_{(X)} ; f(0) \in D_{M^{\prime}}^{\prime}\right\},\left(E\left[\operatorname{Int}\left(R_{N}\right)\right]\right)^{\prime} \subseteq V$, and hence the dimension of the Prüfer domain $\left(E\left[\operatorname{Int}\left(R_{N}\right)\right]\right)^{\prime}$, being equal to its valuative dimension, is at least 2 , the desired contradiction.

We are grateful to Professor Paul-Jean Cahen for pointing out to us an error in our original proof of Theorem 2.3.

The construction of the famous examples of Nagata of Noetherian domains $D$ that are not catenary (cf. [Na, pp. 204-205; Mu1, pp. 87-88; ZS, pp. 327329]) start with a ground field $k$, which can be taken to be a finite field. If $k$ is finite, then such a domain $D$ has dimension greater than 1 , the integral closure $D^{\prime}$ of $D$ fails to satisfy the conclusion of Theorem 2.3 , and hence $\operatorname{Int}(D)$ is not Noetherian. These domains have nonzero conductors with respect to their integral closures, so they provide counterexamples to Proposition 3.1 of [ $\mathrm{Ch} 1$ ]; the error in the proof of Proposition 3.1 of [Ch1] is its tacit assumption that each maximal ideal of the integral closure has height greater than one. We note that (3.1) of [Ch1] is used in the appendix of that paper to show that $\operatorname{dim}(\operatorname{Int}(D))=\operatorname{dim}(D)+1$ for a Noetherian domain $D$; a proof for this latter statement independent of [Chl, (3.1)] is given in [Ca1], where Corollary 1.3 asserts validity of the equality for a Jaffard domain $D$.

From Theorem 2.3 we have the following corollaries for one-dimensional domains and normal domains: 
Corollary 2.4. For $D$ a one-dimensional Noetherian domain, the following statements are equivalent:

(1) $\operatorname{Int}(D)$ is Noetherian.

(2) $\operatorname{Int}(D)=D[X]$.

(3) The residue field at each maximal ideal of $D$ is infinite.

Proof. (3) $\Rightarrow(2)$ : Apply Fact 1.1 .

$(2) \Rightarrow(1)$ : Clear.

$(1) \Rightarrow(3)$ : Apply Theorem 2.3.

Corollary 2.5. For D an integrally closed Noetherian domain, the following statements are equivalent:

(1) $\operatorname{Int}(D)$ is Noetherian.

(2) $\operatorname{Int}(D)=D[X]$.

(3) $D / P$ is infinite for each height-one prime ideal $P$ of $D$.

\section{The Noetherian PROPERTY IN $\operatorname{Spec}(\operatorname{Int}(D))$}

Suppose that $(D, M)$ is a one-dimensional local domain for which $D / M$ is finite, and let $E=\operatorname{Int}(D)$. It is natural to ask for conditions under which $\operatorname{Spec}(E)$ is Noetherian. In [Ch1] it was shown that for such a $D, \operatorname{Spec}(E)$ consists of height-one primes $f(X) K[X] \cap E$, where $f(X)$ is irreducible in $K[X]$, and maximal ideals of the form $M_{a}=\{f(X) \in E: f(a) \in \widehat{M}\}$, where $a$ varies over the completion $\widehat{D}$ of $D$ and $\widehat{M}=M \widehat{D}$ is the maximal ideal of $\widehat{D}$ (the ideals $M_{a}$ are distinct only if $\widehat{D}$ is an integral domain [CGH; Ch3]). The primes contracted from $K[X]$ form a Noetherian space, so $\operatorname{Spec}(E)$ is Noetherian iff the maximal ideals $M_{a}$ form a Noetherian space; moreover, since the ideals $M_{a}$ are precisely the minimal primes of $M E$, this is true iff they are finite in number-i.e., iff $\operatorname{Spec}(E / M E)$ is finite. Suppose $D^{\prime}$ is a finitely generated $D$-module. Since $\widehat{D}$ is an integral domain iff $D^{\prime}$ is local and a finitely generated $D$-module [Na, Exercise 1, p. 122], if $D^{\prime}$ is local, then it follows from the result in [CGH or Ch3] cited above that $\operatorname{Spec}(E / M E)$ is infinite and hence $\operatorname{Spec}(E)$ is not Noetherian. On the other hand, if $D^{\prime}$ has more than one maximal ideal, then Cahen [Ca2, Lemme 1.2] shows that for $a, b$ in $D$, the ideals $M_{a}, M_{b}$ can be distinct only if $a, b$ represent different cosets in $D$ of the nonzero conductor $C$ of $D^{\prime}$ into $D$. If $f \in M_{a}$, then $f(a+C) \subseteq M$, and hence, by continuity, $f(a+C \widehat{D}) \subseteq \widehat{M}$. Since $\widehat{D}=D+C \widehat{D}$, it follows that if the elements $u, v$ of $\widehat{D}$ belong to the same coset of $C \widehat{D}$, then $M_{u}=M_{v}$. Because $\widehat{D} / C \widehat{D} \cong D / C$ is finite, then $\operatorname{Spec}(E / M E)$ is finite in this case and $\operatorname{Spec}(E)$ is Noetherian. The following result modifies the proofs of these results only slightly to determine whether $\operatorname{Spec}(E)$ is Noetherian in the case where $D^{\prime}$ is not a finitely generated $D$-module.

Theorem 3.1. Let $(D, M)$ be a one-dimensional local domain with $D / M$ finite. (1) Suppose $D^{\prime}$ is local (and hence a discrete rank-one valuation domain). If $a, b$ are distinct elements of $D$, then there is an $f(X) \in \operatorname{Int}(D)$ for which $f(a) \notin M$ but $f(b)=0$. In particular, $M_{a} \neq M_{b}$.

(2) Suppose $D^{\prime}$ is not local. Let $R$ be a finite extension of $D$ in $D^{\prime}$ such that the maximal ideals of $D^{\prime}$ have distinct contractions $N_{1}, \ldots, N_{k}$ to $R$, and suppose the positive integer $\alpha$ is such that $\left(N_{1} \cap \cdots \cap N_{k}\right)^{\alpha}$ is contained in the 
(nonzero) conductor of $R$ into $D$. If $a, b$ in $\widehat{D}$ are such that $a-b \in\left(N_{1}^{\alpha} \cap D\right) \widehat{D}$, then $M_{a}=M_{b}$.

Therefore $\operatorname{Spec}(\operatorname{Int}(D))$ is Noetherian iff $D^{\prime}$ has more than one maximal ideal.

Proof. (1) Suppose we have found an element $g(X)$ of $\operatorname{Int}(D)$ for which $g(a-b) \notin M$ and $g(0)=0$; then $f(X)=g(X-b)$ has the desired property. So we may assume $b=0$. Let $T$ be a set of coset representatives of the maximal ideal $M^{\prime}$ in $D^{\prime}$ for which $0 \in T$, and let $d$ be a generator of $M^{\prime}$. Set $f_{1}(X)=(1 / d) \prod\{(X-t): t \in T\}$ and for $k>1$, set $f_{k}(X)=f_{1}\left(f_{k-1}(X)\right)$. Then it is easy to see that, for any positive integer $n$ and any element $c$ of $D^{\prime}$, $f_{1}\left(d^{n} c\right)$ is associate in $D^{\prime}$ to $d^{n-1} c$, so if $n \geq k$, then $f_{k}\left(d^{n} c\right)$ is associate to $d^{n-k} c$. It follows that if $a$ is associate in $D^{\prime}$ to $d^{k}$, then $f_{k}(a)$ is a unit in $D^{\prime}$, but $f_{k}(0)=0$. The same statements hold for any power of $f_{k}(X)$. Now since $f_{k}(D)$ is contained in both $D^{\prime}$ and a finitely generated $D$-submodule of $K$, it is contained in a subring $R$ of $D^{\prime}$ that is finitely generated over $D$; and $R$ is also local, say with maximal ideal $N$. Some power $N^{m}$ of $N$ is contained in the nonzero conductor of $R$ into $D$. Let $q, p$ denote the (finite) cardinality and characteristic, respectively, of $D^{\prime} / M^{\prime}$. Then the $(q-1)$ st power of any element of $R$ differs from either 0 or 1 (which are in $D$ ) by an element of $N$; and since $p \in N$, it follows from a basic property of binomial coefficients that if $n=(q-1) p^{m}$, then the $n$th power of any element of $R$ is in $D$ (cf. [Na, (31.3)]). Thus $f_{k}^{n}(X) \in \operatorname{Int}(D)$.

(2) Note first that since $D^{\prime}$ has only finitely many maximal ideals by the Krull-Akizuki Theorem [Na, (33.2)], one can always find an $R$ with the desired property by adjoining to $D$ one element from each maximal ideal of $D^{\prime}$ that is not in any other maximal ideal.

Assume by way of contradiction that $M_{a} \neq M_{b}$; then there is an element $g(X) \in \operatorname{Int}(D)$ for which $g(a)-g(b) \notin \widehat{M}$. Write $I=N_{1}^{\alpha} \cap D$. Since $g(X)$ is continuous, we can replace $a, b$ with approximating elements $a_{1}, b_{1}$ of $D$ such that $a_{1}-b_{1} \in I$ and $g\left(a_{1}\right)-g\left(b_{1}\right) \notin M$; so we may assume $a, b \in D$. Now suppose the maximal ideals of $D^{\prime}$ are $M_{1}^{\prime}, \ldots, M_{k}^{\prime}$, lying over $N_{1}, \ldots, N_{k}$ respectively, and for $i=1, \ldots, k$, let $v_{i}$ denote the valuation associated to the discrete rank-one valuation domain $D_{M_{i}^{\prime}}^{\prime}$, and let $\gamma_{i}$ be a positive integer such that for $x, y$ in $K$,

$$
v_{i}(x-y)>\gamma_{i} \text { implies } v_{i}(g(x)-g(y))>0 .
$$

(For instance, $\gamma_{i}$ could be greater than the negative of the smallest $v_{i}$-value among those of the coefficients of $g(X)$.) By the Chinese Remainder Theorem, choose $z$ in $R$ such that

$$
z \equiv b \bmod N_{1}^{\max \left\{\alpha, \gamma_{1}\right\}}, \quad z \equiv a \bmod N_{2}^{\max \left\{\alpha, \gamma_{2}\right\}},
$$

and

$$
z \equiv a \bmod N_{i}^{\alpha}, \quad i=3, \ldots, k .
$$

Then $z-a=(z-b)-(a-b) \in N_{1}^{\alpha}$ and $z-a \in N_{i}^{\alpha}$ for $i>1$. Since the $N_{i}^{\alpha}$ are comaximal, their intersection is their product, so $z-a$ is in the conductor of $R$ into $D$, and hence $z \in D$. Thus, $g(z)-g(b) \in M_{1}^{\prime} D_{M_{1}^{\prime}}^{\prime} \cap D=M$ and $g(z)-g(a) \in M_{2}^{\prime} D_{M_{2}^{\prime}}^{\prime} \cap D=M$; but this yields $g(a)-g(b)=(g(z)-g(b))$-$(g(z)-g(a)) \in M$, the desired contradiction. 
It remains to prove the final statement in the theorem: If $D^{\prime}$ is local, then by (1), $\operatorname{Spec}(E / M E)$ has at least the cardinality of $D$ itself, so $\operatorname{Spec}(E / M E)$ is infinite. But if $D^{\prime}$ is not local, then by (2) the cardinality of $\operatorname{Spec}(E / M E)$ is bounded above by the cardinality of $\widehat{D} / I \widehat{D}=D / I[\mathrm{Na},(17.9)]$, a finite ring.

The question of when $\operatorname{Spec}(\operatorname{Int}(D))$ is Noetherian for $D$ not local or of higher dimension than one remains open. But Corollary 4.4 gives an affirmative answer in many cases of higher dimension, since a finitely generated algebra over a ring with Noetherian spectrum has Noetherian spectrum.

Another consequence of (2) above (or, in the case where $D^{\prime}$ is a finitely generated $D$-module, of Lemme 1.2 of [Ca2]) is:

Remark 3.2. Let $(D, M)$ be a one-dimensional local domain with $D / M$ finite and with integral closure $D^{\prime}$ not local. Then the integral closure $E^{\prime}$ of $E=$ $\operatorname{Int}(D)$ is not a finitely generated $E$-module.

Proof. By (2), $\operatorname{Spec}(E / M E)$ is finite; so it is enough to show that $\operatorname{Spec}\left(E^{\prime} / M E^{\prime}\right)$ is infinite, since if $E^{\prime}$ were finitely generated over $E$, then there would be only finitely many prime ideals of $E^{\prime}$ having the same contraction to $E$. (Indeed, for any $P$ in $\operatorname{Spec}(E), E^{\prime} \otimes_{E}\left(E_{P} / P E_{P}\right)$ is a finite-dimensional vector space over $E_{P} / P E_{P}$, so the dimension of $E^{\prime} \otimes_{E}\left(E_{P} / P E_{P}\right)$ is an upper bound for the number of primes of $E^{\prime}$ lying over $P$. Cf. [Mu2, Exercise 9.3, p. 69].) Note first that, because $\operatorname{Int}\left(D^{\prime}\right)$ is a Prüfer domain, all rings between it and its field of fractions $K(X)$ are integrally closed; so $E^{\prime}=E\left[\operatorname{Int}\left(D^{\prime}\right)\right]$. Now suppose $a \in D$, and fix a maximal ideal $M^{\prime}$ of $D^{\prime}$. Then $a \in \widehat{D}$ and $a \in \widehat{D_{M^{\prime}}}$. Thus if $v$ denotes the discrete rank-one valuation associated with $\widehat{D_{M^{\prime}}^{\prime}}$, then the ranktwo valuation domain $V_{a}=\left\{f \in K[X]_{(X-a)}: v(f(a)) \geq 0\right\}$ contains $E$ and $\operatorname{Int}\left(D_{M^{\prime}}^{\prime}\right)$; so $E^{\prime} \subseteq E\left[\operatorname{Int}\left(D_{M^{\prime}}^{\prime}\right)\right] \subseteq V_{a}$. Moreover, if $a, b \in D$ with $a \neq b$, then $V_{a}, V_{b}$ have distinct centers on $\operatorname{Int}\left(D_{M^{\prime}}^{\prime}\right)$ and hence on $\operatorname{Int}\left(D^{\prime}\right)$ and hence on $E^{\prime}$. Since these centers contain $M, \operatorname{Spec}\left(E^{\prime} / M E^{\prime}\right)$ has at least the cardinality of $D$.

Question 3.3. If $D$ is a one-dimensional local domain with finite residue field for which $D<D^{\prime}$, is it possible for $(\operatorname{Int}(D))^{\prime}$ to be a finitely generated $\operatorname{Int}(D)$ module? For example, if $D=k\left[\left[t^{2}, t^{3}\right]\right]$, where $k$ is a finite field and $t$ is an indeterminate, is $(\operatorname{Int}(D))^{\prime}$ a finitely generated $\operatorname{Int}(D)$-module?

\section{The INCLUSION OF $\operatorname{Int}(D)$ IN $D^{\prime}[X]$ AND THE NOETHERIAN PROPERTY REVISITED}

The methods used to prove Fact 1.1 yield a slightly stronger result, which will be useful to us in proving a partial converse to Theorem 2.3.

Proposition 4.1. Let $S$ be a subset of a domain $R, P$ be a prime ideal of $R$, and $f(X)$ be a polynomial with coefficients in the field of fractions of $R$ for which $f(S) \subseteq R$. If the number of cosets of $P$ in $R$ met by $S$ is greater than $\operatorname{deg}(f)$, then $f(X) \in R_{P}[X]$.

Proof. Write $f(X)=f_{0}+f_{1} X+\cdots+f_{n} X^{n}$, and take elements $t_{0}, t_{1}, \ldots, t_{n}$ of $S$ in distinct cosets of $P$. If $T$ denotes the $(n+1) \times(n+1)$ Vandermonde matrix whose $j$ th column is the $(j-1)$ st powers of the elements $t_{i}$ in order, and 
if $F$ is the column matrix of the coefficients $f_{i}$, then $T F$ is the column matrix with entries $f\left(t_{i}\right)$, all in $R$. The determinant of $T$ is $d=\prod_{i<j}\left(t_{i}-t_{j}\right)$, which by our choice of the elements $t_{i}$ is not in $P$. But for each $i=0,1, \ldots, n$, Cramer's rule shows that $d f_{i}$ is the determinant of a matrix with entries in $R$, and hence in $R_{P}$, and since $d$ is a unit in $R_{P}, f_{i} \in R_{P}$.

Definition 4.2. For a domain $D$ that is not a finite field, we set

$$
D^{a}=\bigcap\left\{D_{P}: P \in \operatorname{Spec}(D) \text { such that } D / P \text { is infinite }\right\} .
$$

We note that, for a prime $P$ of $D, D / P$ is infinite iff either $P$ is not maximal or $P$ is maximal but has infinite residue field. The proof of part (1) of the following result is immediate from Proposition 4.1, and part (2) follows from part (1).

Corollary 4.3. Suppose $D$ is a subring of a domain $R$. (1) For each $P$ in $\operatorname{Spec}(R)$ such that $D /(P \cap D)$ is infinite, $\operatorname{Int}(D) \subseteq R_{P}[X]$. In particular, $\operatorname{Int}(D) \subseteq D^{a}[X]$.

(2) If $D$ is Noetherian and $\bigcap\left\{R_{P}: P \in \operatorname{Spec}(R)\right.$ and $D /(P \cap D)$ is infinite $\}$ is a finitely generated D-module, then $\operatorname{Int}(D)$ is Noetherian. In particular, if $D^{a}$ is a finitely generated D-module, then $\operatorname{Int}(D)$ is Noetherian.

Suppose $D$ is Noetherian. Then it follows from [Na, (33.10)] that for a prime ideal $P^{\prime}$ of $D^{\prime}, D^{\prime} / P^{\prime}$ is infinite iff $D /\left(P^{\prime} \cap D\right)$ is infinite, and hence $D^{a} \subseteq\left(D^{\prime}\right)^{a}$. If for every height-one prime $P^{\prime}$ of $D^{\prime}, D^{\prime} / P^{\prime}$ is infinite, then the fact that $D^{\prime}$ is Krull implies that

$$
\left(D^{\prime}\right)^{a} \subseteq \bigcap\left\{D_{P^{\prime}}^{\prime}: P^{\prime} \text { is a height-one prime of } D^{\prime}\right\}=D^{\prime},
$$

so that $\left(D^{\prime}\right)^{a}=D^{\prime}$. These observations and Corollary 4.3 then yield the following result.

Corollary 4.4. Let $D$ be a Noetherian domain such that for every height-one prime $P^{\prime}$ of the integral closure $D^{\prime}$ of $D, D^{\prime} / P^{\prime}$ is infinite (for example, such that $D^{\prime}$ has no height-one maximal ideals). Then $\operatorname{Int}(D) \subseteq D^{\prime}[X]$. In particular, in this case, if $D^{\prime}$ is a finitely generated $D$-module, then $\operatorname{Int}(D)$ is Noetherian.

A familiar domain to which this corollary applies is constructed as follows: Let $u, v$ be indeterminates over the finite field $k$, and set $D=k[[u, v]]$. Write $D^{\prime}$ in the form $D^{\prime}=k+M$, where $M=(u, v) k[[u, v]]$ is the unique maximal ideal of $D^{\prime}$, and set $D=k+M^{2}$. Then the unique maximal ideal $M^{2}$ of $D$ is an associated prime of a principal ideal (for example, $M^{2}=$ $\left.\left(u^{2} D: u^{2} v\right)\right)$, so $\operatorname{Int}(D)>D[X]$. But since the conductor of $D^{\prime}$ into $D$ is nonzero (in fact, the conductor is $M^{2}$ ), $D^{\prime}$ is a finitely generated $D$-module, so $\operatorname{Int}(D)$ is Noetherian. This shows that Corollary 2.4 does not extend to higher dimensional domains $D$.

In [Mj], Matijevic defined the "global transform" of a Noetherian domain $D$ as follows:

$$
D^{g}=\{x \in K: \exists I \text { an ideal of } D \text { such that } \operatorname{dim}(D / I)=0 \text { and } x I \subseteq D\} .
$$

In other words, $D^{g}$ is the directed union of the transforms of all finite products of maximal ideals of $D$. For $D$ not a field, this definition is equivalent to

$$
D^{g}=\bigcap\left\{D_{P}: P \in \operatorname{Spec}(D) \backslash \operatorname{Mspec}(D)\right\} .
$$


Thus, for a Noetherian domain $D, D^{a}$ is contained in $D^{g}$. Hence, by Corollary 4.3 , if $D^{g}$ is a finitely generated $D$-module, then $\operatorname{Int}(D)$ is Noetherian. Nishimura [Ni, Proposition 1.2] shows that if every maximal ideal of the integral closure $D_{D \backslash M}^{\prime}$ of $D_{M}$ has height greater than one, the $M D_{M}$-transform $\bigcap\left\{D_{P}: P \in \operatorname{Spec}(D), P<M\right\}$ of $D_{M}$ is integral over $D_{M}$-i.e., is contained in $D_{D \backslash M}^{\prime}$. Taking intersection as $M$ varies over $\operatorname{Mspec}(D)$, we conclude that

$$
\operatorname{Int}(D) \subseteq D^{a}[X] \subseteq D^{g}[X] \subseteq D^{\prime}[X] .
$$

Thus, we obtain an alternative proof of Corollary 4.4. Ferrand and Raynaud [FR, Proposition 3.3 and Remarques 3.7] give examples of two-dimensional local domains $(D, M)$ for which the transform $D^{g}$ of $M$ is not a finitely generated $D$-module, but in these examples $D / M$ is infinite. It seems possible that there exist examples of the type constructed by Ferrand and Raynaud but with finite residue field. So the question of whether, for a local domain $D$ of dimension greater than $1, \operatorname{Int}(D)$ is Noetherian $\operatorname{iff} \operatorname{Int}(D)$ is a finitely generated $D$-algebra remains open.

\section{Locally Noetherian does NOT IMPly NoETHERIAN}

Our next example shows that $\operatorname{Int}(D)$ may be locally Noetherian in a strong sense without being Noetherian. The final touch in the example is provided by the following lemma.

Lemma 5.1. Let $(D, M)$ be a local domain with finite residue field and integral closure $D^{\prime}$ having no height-one maximal ideals. Assume that $M$ is an associated prime of a principal ideal of $D$. Then for some maximal ideal $M^{\prime}$ of $D^{\prime},\left(M^{\prime}, X\right) D^{\prime}[X] \cap \operatorname{Int}(D)=P$ is an associated prime of $(X) \operatorname{Int}(D)$, and $P \cap D=M$.

Proof. Since $M$ has the form $d D:_{D} c$ for some elements $d, c$ of $D$, if we set $b=c / d$, we get $M=D_{:_{D}} b$. Let $T$ be a set of coset representatives of $M$ in $D$ such that $0 \in T$, and set $f(X)=\prod\{(X-t): t \in T\}$. Then $b f(X) \in \operatorname{Int}(D) \backslash D[X]$ and $b f(X) / X \notin \operatorname{Int}(D)$, so $(X) \operatorname{Int}(D):_{\operatorname{In}(D)} b f(X)$ is a proper ideal of $\operatorname{Int}(D)$. Since $(M, X) D[X]$ is maximal, it is contained in only maximal ideals of $D^{\prime}[X]$, of the form $\left(M^{\prime}, X\right) D^{\prime}[X]$. But $(M, X) \operatorname{Int}(D) \subseteq$ $(X) \operatorname{Int}(D): \ln (D) b f(X)$, so at least one of the minimal primes $P$ of $(M, X) \operatorname{Int}(D)$ is an associated prime of $(X) \operatorname{Int}(D)$.

Example 5.2. A two-dimensional Noetherian domain $D$ such that $\operatorname{Int}(D)$ is not Noetherian but $\operatorname{Int}\left(D_{M}\right)$ is Noetherian for each maximal ideal $M$ of $D$ : Let $k$ be a finite field, $p$ be its characteristic, and $u, v$ be indeterminates. Enumerate the nonzero elements of $k[u, v]: g_{1}, g_{2}, \ldots$. For each positive integer $i$ in turn: Pick a maximal ideal $N_{i}$ of $k[u, v]$ such that $b_{1} \cdots b_{i-1} g_{1} \cdots g_{i} \notin N_{i}$. (This is possible because $k[u, v]$ is a Hilbert ring. The elements $b_{j}$ will be selected below; there are no $b_{j}$ in the product avoided to select $N_{1}$.) Let $S_{i}$ denote the localization of $k[u, v]$ at $N_{i}$, a two-dimensional regular local ring, and pick $a_{i}, b_{i} \in k[u, v]$ such that $b_{i} \in N_{i} \backslash\left(N_{i}^{2} \cup N_{1} \cup \cdots \cup N_{i-1}\right)$ and $a_{i}, b_{i}$ is a minimal generating set for $N_{i} S_{i}$. Let $R_{i}$ denote the localization of $S_{i}\left[\left(a_{i} / b_{i}\right)^{2},\left(a_{i} / b_{i}\right)^{3}\right]$ at its maximal ideal $\left(a_{i}, b_{i},\left(a_{i} / b_{i}\right)^{2},\left(a_{i} / b_{i}\right)^{3}\right)$, so that the integral closure $R_{i}^{\prime}$ of $R_{i}$ is the localization of $S_{i}\left[a_{i} / b_{i}\right]$ at its maximal ideal $\left(a_{i} / b_{i}, b_{i}\right)$. Set $D=\bigcap_{i=1}^{\infty} R_{i}$; then since for any $f$ in $R_{i}^{\prime}, f^{p} \in R_{i}$, we 
see that $D^{\prime}=\bigcap_{i=1}^{\infty} R_{i}^{\prime}$ is integral over $D$. Also $D^{\prime}$ is contained in the field of fractions $k(u, v)$ of $D$ and is integrally closed, so $D^{\prime}$ is indeed the integral closure of $D$.

Next we note that $\left\{R_{i}\right\}_{i=1}^{\infty}$ and $\left\{R_{i}^{\prime}\right\}_{i=1}^{\infty}$ are locally finite families, i.e., that any nonzero element of $k(u, v)$ is a unit in all but finitely many of each family. Since $k[u, v] \subseteq R_{i} \subseteq R_{i}^{\prime}$, it is enough to note that, by our choice of the $N_{i}$, no nonzero element $g_{j}$ of $k[u, v]$ is contained in infinitely many of the centers $N_{i}$ of $R_{i}$ and $R_{i}^{\prime}$ on $k[u, v]$. The property of local finiteness allows us to "commute localization and intersection," i.e., for any multiplicatively closed subset $T$ of $D, D_{T}=\bigcap_{i=1}^{\infty}\left(R_{i}\right)_{T}$ and similarly for $D^{\prime}$. In particular, if $T=$ $k[u, v] \backslash N_{i}$, then for any $j$ distinct from $i$, since $b_{j} \notin N_{i},\left(R_{j}\right)_{T}=\left(R_{j}^{\prime}\right)_{T} \supseteq$ $\left(S_{j}\right)_{T}=\bigcap\left\{k[u, v]_{P}: P \in \operatorname{Spec}(k[u, v]), P \subseteq N_{i} \cap N_{j}\right\} \supseteq R_{i}^{\prime} \supseteq R_{i}$, while $\left(R_{i}\right)_{T}=R_{i}$; so if $M_{i}$ is the center on $D$ of the maximal ideal of $R_{i}$, we get $R_{i}=D_{T} \subseteq D_{M_{i}} \subseteq R_{i}$, so $D_{M_{i}}=R_{i}$. Similarly if $M_{i}^{\prime}$ denotes the center on $D^{\prime}$ of the maximal ideal of $R_{i}^{\prime}$, then $\left(D^{\prime}\right)_{M_{i}^{\prime}}=R_{i}^{\prime}$.

Now we want to show that $D$ is Noetherian. Let $d$ be a nonzero element of $D$; then $d D=\bigcap_{i=1}^{\infty} d R_{i}$, and $d R_{i}=R_{i}$ for all but finitely many positive integers $i$, so $d D$ is the intersection of finitely many primary ideals (contracted from the remaining $R_{i}$ ). So to see that $D$ is Noetherian, it suffices to show that, for each nonzero primary ideal $Q$ of $D, D / Q$ is Noetherian.

Suppose first that the radical $P$ of $Q$ is a height-one prime of $D$. Then $P$ does not contain any $M_{i}$, so $\left(R_{i}\right)_{D \backslash P}$ is a proper localization of $R_{i}$, i.e., one of $a_{i}, b_{i},\left(a_{i} / b_{i}\right)^{2},\left(a_{i} / b_{i}\right)^{3}$ is a unit in $\left(R_{i}\right)_{D \backslash P}$. It is easy to check in each of these four cases that $a_{i} / b_{i} \in\left(R_{i}\right)_{D \backslash P}$, and hence $\left(R_{i}\right)_{D \backslash P}=\left(R_{i}^{\prime}\right)_{D \backslash P}$. Now the height-one primes of $R_{i}^{\prime}$ are $b_{i} R_{i}^{\prime}$ and some of the form $(f) k[u, v]_{(f)} \cap R_{i}^{\prime}$ for $f$ irreducible in $k[u, v]$. Since $b_{i} R_{i}^{\prime}$ lies over $N_{i}$ in $k[u, v]$, the localization of $R_{i}^{\prime}$ at $b_{i} R_{i}^{\prime}$ is a discrete rank-one valuation domain containing $k[u, v]$, and by our choice of the $N_{i}$ these localizations form a locally finite family. The localizations of $k[u, v]$ at height-one primes also form a locally finite family, so the intersection of any subfamily of the union of these two families is a Krull domain. In particular, $D_{P}=\bigcap_{i=1}^{\infty}\left(R_{i}\right)_{D \backslash P}=\bigcap_{i=1}^{\infty}\left(R_{i}^{\prime}\right)_{D \backslash P}$ is Krull, and since $D_{P}$ is also one-dimensional and local, it is a discrete rank-one valuation domain. Thus $Q$ is a symbolic power of $P$. Moreover, $D / P$ is Noetherian: If $P=(f) k[u, v]_{(f)} \cap D$, then $D / P$ lies between $k[u, v] /(f)$ and its field of fractions, so by the Krull-Akizuki Theorem $D / P$ is Noetherian. If $P=b_{i} R_{i}^{\prime} \cap D$ for some $i$, then $D / P$ lies between the field $F=k[u, v] / N_{i}$ and $R_{i}^{\prime} / b_{i} R_{i}^{\prime}$, a polynomial ring in one indeterminate over $F$; so again $D / P$ is Noetherian. It follows from [HL, Lemma 3.3] that $D / Q$ is Noetherian in this case.

Now suppose the radical $M$ of $Q$ is maximal in $D$. Then $D / Q=D_{M} / Q D_{M}$, so it is enough to show that $D_{M}$ is Noetherian. For $M=M_{i}, D_{M}=R_{i}$ is Noetherian. If $M \neq M_{i}$ for every $i$, then $D_{M}=\bigcap_{i=1}^{\infty}\left(R_{i}\right)_{D \backslash M}=\bigcap_{i=1}^{\infty}\left(R_{i}^{\prime}\right)_{D \backslash M}$ is a Krull domain between $k[u, v]$ and its field of fractions; so it is Noetherian by [H, Theorem 9].

We can also use the descriptions of the localizations of $D$ at its maximal ideals $M$ in the last paragraph to see that $\operatorname{Int}\left(D_{M}\right)$ is Noetherian: If $M=M_{i}$, then $\operatorname{Int}\left(D_{M}\right)=\operatorname{Int}\left(R_{i}\right)$, which lies between $R_{i}[X]$ and $R_{i}^{\prime}[X]$; since $R_{i}^{\prime}$ has nonzero conductor into $R_{i}$, we see that $\operatorname{Int}\left(D_{M}\right)$ is Noetherian. Suppose $M \neq$ $M_{i}$ for every $i$; then as we saw above, $D_{M}$ is integrally closed. Moreover, 
if $M$ were of height one, then $D_{M}$ would be a discrete rank-one valuation domain and hence a localization of $D^{\prime}$, i.e., a localization of one of the $R_{i}^{\prime}$ at a height-one prime, i.e., a localization of one of the $R_{i}=D_{M_{i}}$, which would imply that $M \subseteq M_{i}$. It follows that $M$ has height two, and hence by Corollary 2.5 that $\operatorname{Int}\left(D_{M}\right)=D_{M}[X]$, which is Noetherian.

Finally, assume by way of contradiction that $\operatorname{Int}(D)$ is Noetherian. For each positive integer $i, M_{i} R_{i}=\left(a_{i} / b_{i}\right)^{2} R_{i}:\left(a_{i} / b_{i}\right)^{3}$ is an associated prime of $\left(a_{i} / b_{i}\right)^{2} R_{i}$; so by Lemma 5.1, $\left(M_{i}^{\prime}, X\right) R_{i}^{\prime}[X] \cap \operatorname{Int}\left(R_{i}\right)=P_{i}$ is an associated prime of $(X)$ in $\operatorname{Int}\left(R_{i}\right)$, and hence $P_{i} \cap \operatorname{Int}(D)$ is an associated prime of $(X)$ in $\operatorname{Int}(D)$. All the $P_{i} \cap \operatorname{Int}(D)$ are distinct (since their intersections with $D$ are the distinct $\left.M_{i}\right)$, so $(X)$ has infinitely many associated primes in $\operatorname{Int}(D)$, the desired contradiction. Thus, $\operatorname{Int}(D)$ is not Noetherian.

\section{The INCLusion of $\operatorname{Int}(D)$ IN $\operatorname{Int}\left(D^{\prime}\right)$}

In conclusion we provide a description of the one-dimensional Noetherian domains $D$ for which $\operatorname{Int}(D) \subseteq \operatorname{Int}\left(D^{\prime}\right)$.

Proposition 6.1. Let $D$ be a one-dimensional Noetherian domain with integral closure $D^{\prime}$. The following conditions are equivalent:

(1) $\operatorname{Int}(D) \subseteq \operatorname{Int}\left(D^{\prime}\right)$.

(2) For each maximal ideal $M^{\prime}$ of $D^{\prime}$ for which $D^{\prime} / M^{\prime}$ is finite, we have $D^{\prime} / M^{\prime}=D /\left(M^{\prime} \cap D\right)$ and $\left(M^{\prime} \cap D\right) D_{M^{\prime}}^{\prime}=M^{\prime} D_{M^{\prime}}^{\prime}$.

Proof. It is clear that (2) is a local statement; since $D$ and $D^{\prime}$ are Noetherian, it follows easily from [SSY, Proposition 5] that (1) is also local-i.e., that $\operatorname{Int}(D) \subseteq$ $\operatorname{Int}\left(D^{\prime}\right)$ iff $\operatorname{Int}\left(D_{M^{\prime} \cap D}\right) \subseteq \operatorname{Int}\left(D_{M^{\prime}}^{\prime}\right)$ for every maximal ideal $M^{\prime}$ of $D^{\prime}$. Thus we may assume that $D$ is local with maximal ideal $M$ (so that $D^{\prime}$ is a semilocal principal ideal domain) and consider a particular maximal ideal $M^{\prime}$ of $D^{\prime}$. Moreover, if $D^{\prime} / M^{\prime}$ is infinite, then $D / M$ is also infinite, so $\operatorname{Int}(D)=D[X] \subseteq$ $D_{M^{\prime}}^{\prime}[X]=\operatorname{Int}\left(D_{M^{\prime}}^{\prime}\right)$; thus we need only consider those $M^{\prime}$ for which the residue field is finite.

(2) $\Rightarrow(1)$. Let $R=D_{M^{\prime}}^{\prime}$; then the hypotheses of (2) imply that $R=$ $D+M R$. Take $f(X) \in \operatorname{Int}(D)$, and write $f(X)=g(X) / d$, where $g(X) \in D[X]$ and $d \in D \backslash(0)$. Choose a power $M^{n}$ of $M$ for which $M^{n} \subseteq d D$. Then

$$
R=D+M R=D+M(D+M R)=D+M^{2} R=\cdots=D+M^{n} R=D+d R .
$$

Thus, for any element $r$ of $R$ there is an element $a$ in $D$ such that $r-a \in d R$; and since $g(X) \in D[X] \subseteq R[X]$, we have $g(r)-g(a)$ is divisible in $R$ by $r-a$ and hence by $d$. Thus, $g(r)=d(f(a))+(g(r)-g(a)) \in d R$-i.e., $f(r) \in R$. We conclude that $f(X) \in \operatorname{Int}(R)=\operatorname{Int}\left(D_{M^{\prime}}^{\prime}\right)$.

(1) $\Rightarrow(2)$. Let $T$ be a set of coset representatives of $D$ in $M$ such that $0 \in T$, and let $p(X)=\prod\{X-t: t \in T\}$. If $D / M<D^{\prime} / M^{\prime}$, then there exists an element $b$ of $D^{\prime}$ that is congruent to no element of $T$ modulo $M^{\prime}$. Choose $m$ in $M \backslash(0)$ and a positive integer $k$ such that $M^{k} \subseteq m D$. Then $f(X)=p(X)^{k} / m \in \operatorname{Int}(D)$; but $p(b)^{k} / m \notin D^{\prime}$ because, by the choice of $b, p(b) \notin M^{\prime}$, and hence $p(b)^{k} \notin M^{\prime} \supseteq m D^{\prime}$. Therefore $f(X) \notin \operatorname{Int}\left(D^{\prime}\right)$, and $\operatorname{Int}(D) \nsubseteq \operatorname{Int}\left(D^{\prime}\right)$ in this case. On the other hand, if $D / M=D^{\prime} / M^{\prime}$ but $M D_{M^{\prime}}^{\prime}<M^{\prime} D_{M^{\prime}}^{\prime}$, then $M D_{M^{\prime}}^{\prime} \subseteq\left[M^{\prime} D_{M^{\prime}}^{\prime}\right]^{2}=a^{2} D_{M^{\prime}}^{\prime}$, where $a$ is a generator of $M^{\prime}$ in $D^{\prime}$. Thus if $g(X)=p(X)^{2} / a^{3}$, then $g(D)$ is contained in the Jacobson radical of $D^{\prime}$. As in the proof of Proposition 2.2, it then follows that 
$g(X)^{m} \in \operatorname{Int}(D)$ for some positive integer $m$. However, $g(X)^{m} \notin \operatorname{Int}\left(D^{\prime}\right)$ because, for example, $g(a)^{m} \notin D_{M^{\prime}}^{\prime}$ for any positive integer $m$. This completes the proof.

\section{ACKNOWLEDGMENT}

We are grateful to Professor Paul-Jean Cahen for illuminating and helpful comments. In a recent paper (Polynômes à valeurs entières sur une partie) he has proved a sharper version of Corollary 4.4 , including a converse, and several other results related to those in the present paper.

\section{ADDENDUM}

We remark that in Theorem 6 and Corollary 7 of [GHLS], the hypothesis that the domain $D$ be countable can be replaced by the hypothesis that the spectrum of $D$ is countable. Thus, for example, Corollary 7 of [GHLS] applies in the case where $D$ is a complete discrete rank-one valuation domain.

\section{REFERENCES}

[B] D. Brizolis, A theorem on ideals in Prüfer rings of integral-valued polynomials, Comm. Algebra 7 (1979), 1065-1077.

[Ca1] P.-J. Cahen, Dimension de l'anneau des polynômes a valeurs entières, Manuscripta Math. 67 (1990), 333-343.

[Ca2] _ Polynômes á valeurs entières sur un anneau non analytiquement irreductible, preprint.

[CC] P.-J. Cahen and J.-L. Chabert, Coefficients et valeurs d'un polynôme, Bull. Sci. Math. 95 (1971), 295-304.

[CGH] P.-J. Cahen, F. Grazzini, and Y. Haouat, Intégrité du complété et théorème de StoneWeierstrass, Ann. Sci. Univ. Clermont-Ferrand II Math. 21 (1982), 47-58.

[Ch1] J.-L. Chabert, Les idéaux premiers de l'anneau des polynômes à valeurs entières, J. Reine Angew. Math. 293/294 (1977), 275-283.

[Ch2] _ Un anneau de Prüfer, J. Algebra 107 (1987), 1-16.

[Ch3] _ Le théorème de Stone-Weierstrass et les polynòmes à valeurs entières, Actes du colloque d'algèbre de Montpellier, 1988, (to appear).

[FR] D. Ferrand and M. Raynaud, Fibres formelles d'un anneau noethérien, Ann. Sci. École Norm. Sup. (4) 3 (1970), 295-311.

[G] R. Gilmer, Prüfer domains and rings of integer-valued polynomials, J. Algebra 129 (1990), 502-517.

[GHLS] R. Gilmer, W. Heinzer D. Lantz, and W. Smith, The ring of integer-valued polynomials of a Dedekind domain, Proc. Amer. Math. Soc. 108 (1990), 673-681.

[H] W. Heinzer On Krull overrings of a Noetherian domain, Proc. Amer. Math. Soc. 22 (1969), 217-222.

[HL] W. Heinzer, and D. Lantz, When is an N-ring Noetherian?, J. Pure Appl. Algebra 39 (1986), 125-139.

[Mj] J. Matijevic, Maximal ideal transforms of Noetherian rings, Proc. Amer. Math. Soc. 54 (1976), 49-52.

[Mu1] H. Matsumura, Commutative algebra, 2nd ed., Benjamin/Cummings, Reading, Mass., 1980.

[Mu2] _ Commutative ring theory, Cambridge Univ. Press, Cambridge, 1986.

[Na] M. Nagata, Local rings, Interscience, New York, London and Sydney, 1962.

[Ni] J. Nishimura, On ideal transforms of noetherian rings. I, J. Math. Kyoto Univ. 19 (1979). 41-46. 
[O] A. Ostrowski, Über ganzwertige Polynome in algebraische Zahlkörpern, J. Reine Angew. Math. 358 (1919), 117-124.

[P] G. Pólya, Über ganzwertige Polynome in algebraische Zahlkörpern, J. Reine Math. 358 (1919), 97-116.

[SSY] F. Shibata, T. Sugatani, and K. Yoshida, Note on rings of integral-valued polynomials, C. R. Math. Rep. Acad. Sci. Canada 8 (1986), 297-301.

[ZS] O. Zariski and P. Samuel, Commutative algebra, vol. I, Springer-Verlag, Berlin, 1975.

Department of Mathematics B154, Florida State University, Tallahassee, Florida 32306

E-mail address: gilmer@math.fsu.edu

Department of Mathematics, Purdue University, West Lafayette, Indiana 47907-1395

E-mail address: heinzer@math.purdue.edu

Department of Mathematics, Colgate University, Hamilton, New York 13346-1398

E-mail address: dlantz@colgateu.bitnet 